\title{
La place d'Ottawa-Gatineau parmi les villes du savoir nord-américaines
}

\author{
Rémy Tremblay ${ }^{1}$ \\ INRS-Urbanisation, culture et société
}

\section{Introduction}

Les classements (rankings) intégrés des villes du savoir nord-américaines sont rares. Le seul dont nous avons connaissance est de Gertler ${ }^{2}$. La plupart des classements portent soit sur les villes américaines, soit sur les villes canadiennes ${ }^{3}$. À partir d'un univers des 90 régions métropolitaines (MSA/CMSA américaines et RMR canadiennes) de plus de 500000 habitants (en 2000-2001), nous tenterons, dans cet article, de situer Ottawa (incluant Gatineau) par rapport aux autres villes des États-Unis et du Canada. Pour ce faire, nous utiliserons la méthode du benchmarking, c'est-àdire que nous comparerons Ottawa par le biais de classements reposant sur sept indicateurs qui, en principe, traduisent la force et le potentiel d'une ville dans l'économie du savoir.

Cette étude exploratoire permettra aussi de voir comment Ottawa, reconnue par certains gens d'affaires de cette région comme étant la «Sillicon Valley du Nord », se compare avec ses consœurs nord-américaines.

Avant de présenter nos résultats de recherche exploratoire, nous aimerions nous arrêter brièvement sur la tradition des classements relatifs aux villes du savoir aux États-Unis.

\section{La tradition des rankings et l'économie du savoir}

Parmi les exercices les plus récents de ranking, certains mettent l'accent sur le concept de « créativité ", lequel est fort élastique ${ }^{4}$. Cependant, peu importe les auteurs, le capital humain, les connaissances et la dynamique d'innovation propres aux villes restent au cœur des écrits sur l'économie du savoir ${ }^{5}$, et le secteur tertiaire y occupe désormais une place prépondérante. La présence d'usines et d'industries lourdes seraient dorénavant moins importante qu'une maind'œuvre qualifiée, une bonne qualité de vie et, surtout, la capacité d'attirer et de garder les meilleurs talents ${ }^{6}$. L'histoire récente des villes du Rust Belt aux États-Unis illustre bien le déclin de l'industrie lourde comme base de prospérité. Détroit, à titre d'exemple, qui a connu de sérieux problèmes sociaux et économiques, a réussi à freiner sa descente aux enfers en misant sur des secteurs d'activités tertiaires ${ }^{7}$. En contrepartie, Ottawa, ville de services, a diversifié avec succès son économie, pourtant solide, en promouvant la recherche et le développement de la haute technologie ${ }^{8}$.

Dans une analyse de classements de ce que Tremblay ${ }^{9}$ appelle les « technopoles » nord-américaines, il ressort clairement que la haute technologie, tout comme l'économie du savoir, est un concept qui emprunte une multitude de définitions. Comme l'indiquent si bien Miller et Adams ${ }^{10}$ : " From running shoes to aerospace, everything that could conceivably be so designated gets the high tech label ».

Des six classements analysés par Tremblay, cinq portaient sur des villes américaines seulement. Ces classements provenaient de l'American Electronics Association et NASDAQ ${ }^{11}$, de la Brooking Institute ${ }^{12}$, de la Milken Institute ${ }^{13}$, de la Progressive Policy Institute ${ }^{14}$ et de l'Université du Minnesota ${ }^{15}$. 
À la lumière de cette analyse de différents exercices de classement, quatre conclusions se dégagent :

1. Ce qui distingue les définitions de la haute technologie les unes des autres sont les classes industrielles choisies. Ces classes proviennent de systèmes nationaux de classements industriels tels que les SIC, CTI et SCIAN. Ainsi, une vision plus large de la haute technologie incorpore des classes comme l'aéronautique, la pharmaceutique, la biotechnologie, les services, etc., alors qu'une approche plus réduite se limite aux secteurs se rapportant à la R\&D et à la fabrication de matériel informatique.

2. Le nombre d'employés constitue la principale unité de mesure pour établir l'importance des classes industrielles retenues. Cependant, la valeur de la production (valeur ajoutée) est parfois utilisée.

3. L'univers des villes sélectionnées varie considérablement, et ce autant au niveau de la définition géographique que du nombre. Pour ce qui est de la définition des villes, certains auteurs choisissent les MSA ou CMSA, alors que d'autres préfèrent les PMSA. Au chapitre du nombre de villes choisies, l'univers varie entre 14 et 315 .

4. Selon la méthode de calcul (type d'indice, etc.) et/ou d'unité de mesure (codes SIC sélectionnés, etc.), la position d'une ville donnée peu varier grandement d'un classement à l'autre.

En somme, ce que nous retenons de cette étude de classements est qu'il n'existe pas de système «parfait » de classement, et il ne faut sans doute pas en chercher.

Les problèmes de définition, de standardisation et de disponibilité des données demeurent des obstacles importants, même à l'intérieur d'un seul pays, comme l'illustre la variété des classements américains.

\section{Un « top 20 » nord-américain}

D'abord, le tableau 1 propose une synthèse de nos résultats avec les « top 20 » villes pour chacun des indicateur analysés ci-dessous. Ottawa apparaît en lettres majuscules.

\section{Le taux d'homicides ${ }^{16}$}

Les villes canadiennes dominent largement les « top $20 »$ pour le plus bas taux d'homicides par 1000 habitants, avec Ottawa qui se classe au $8^{\mathrm{e}}$ rang. La criminalité est clairement influencée par des facteurs institutionnels et culturels qui n'ont pas forcément de lien avec la taille de la ville. Quant aux villes américaines les plus sécuritaires, on observe qu'elles se trouvent souvent dans le nord, à quelques heures de route de la frontière canadienne : Grand Rapids (Michigan), Portland (Oregon), Albany, Boston, Minneapolis, Springfield (Massachusetts.) et Seattle.

Les villes canadiennes dominent largement les « top 20 » pour le plus bas taux d'homicides par 1000 habitants, avec Ottawa qui se classe au $8^{\mathrm{e}}$ rang.

\section{Les diplômés universitaires par 1000 habitants ${ }^{17}$}

On y retrouve, parmi les villes du « top $20 »$, celles de taille moyenne comme Austin, Raleigh et Hartford. Ottawa, au $13^{\mathrm{e}}$ rang, est la seule ville canadienne à s'y classer. D'autre part, ce n'est pas une surprise d'y voir les deux grands pôles intellectuels et technologiques de San Francisco et de Boston. Trois facteurs semblent également expliquer la bonne performance des villes de ce classement: être une capitale, être une ville universitaire et être un lieu de retraite.

\section{Les détenteurs de doctorat (Ph.D.) par 1000 habitants ${ }^{18}$}

À nouveau, on remarque que les villes du «top 20 » sont souvent des capitales (12 d'entres elles), des villes universitaires ou des centres de recherche, dont certains reliés à la défense. Ottawa se classe très favorablement, soit au $6^{\mathrm{e}}$ rang.

\section{Les doctorats décernés en sciences et génie par 1000 habitants ${ }^{19}$}

Un premier constat que l'on retire de ce classement est que 9 des 20 villes se trouvaient également dans la colonne portant sur l'indicateur précédant. De plus, ces 9 villes occupent les 10 premiers rangs du « top $20 »$, avec Raleigh qui domine toujours. On note aussi qu'elles sont toutes des villes où se trouvent des 
universités de recherche. D'autre part, on observe la disparition d'Ottawa mais l'apparition de 5 villes canadiennes. Le classement élevé de la ville de Québec s'explique essentiellement par la présence de l'Université Laval. Quant aux autres villes canadiennes du « top 20 », elles possèdent toutes des facultés de sciences et de génie orientées vers des activités à fort contenu de génie : exploration pétrolière et minière, foresterie, métallurgie, énergie et hydroélectricité.

L'orientation traditionnelle de l'économie canadienne, axée sur les ressources naturelles, explique aussi, bien que partiellement, l'importance des facultés de sciences et de génie dans ses villes. À ce titre, la moins bonne performance de Toronto est plus éton- nante puisque son économie a traditionnellement été moins axée sur l'exploitation des ressources naturelles. Ainsi, les résultats sur cet indicateur doivent s'interpréter avec prudence, car ils se limitent aux diplômes décernés pour une année, ce qui peut cacher des variations annuelles importantes.

Le cas d'Ottawa ( $30^{\mathrm{e}}$ parmi les 90 villes analysées) témoigne bien de ce bémol : comme les données de Statistique Canada datent de 1998, elles ne tiennent pas compte de l'apparition depuis les cinq dernières années dans les universités et collèges d'OttawaGatineau de programmes d'études et de recherche en sciences et en génie, incluant plusieurs de niveau doctorale.

Tableau 1 - Les « top 20 »

\begin{tabular}{|c|c|c|c|c|c|c|c|}
\hline Rang & $\begin{array}{c}1 \\
\text { Taux } \\
\text { d'homicides } \\
\text { les plus bas }\end{array}$ & $\begin{array}{l}\text { Diplômés } \\
\text { par } 1000\end{array}$ & $\begin{array}{l}\text { Doctorats } \\
\text { par } 1000\end{array}$ & $\begin{array}{c}\text { Doctorats } \\
\text { S\&G par } 1000\end{array}$ & $\begin{array}{c}\% \text { Serv prof, } \\
\text { sc. et techmques }\end{array}$ & $\begin{array}{l}\% \text { Industrie de } \\
\text { l'information }\end{array}$ & $\begin{array}{l}\text { \% Trafic aérien } \\
\text { international }\end{array}$ \\
\hline 1 & Cleveland & San Francis & Raleigh & Raleigh & Wash-Balt & Denver & Miami \\
\hline 2 & Kansas City & Raleigh & Wash-Balt & Austin & San Francis & Kansas City & New York \\
\hline 3 & Charlotte & Wash-Balt & San Francis & Baton Rouge & Calgary & Atlanta & Honolulu \\
\hline 5 & Scranton & Denver & Austin & Columbus & Toronto & New York & Boston \\
\hline 6 & Youngstown & Austin & OTTAWA & Boston & Raleigh & Dallas & San Francis \\
\hline 7 & Charleston & Minneapolis & Albuquerque & Albany & Denver & San Francis & Houston \\
\hline 8 & OTTAWA & Seattle & Tucson & Québec & Boston & Colorado Spr & Chicago \\
\hline 12 & Hamilton & Hartford & Knoxville & Buffalo & Austin & Seattle & Minneapolis \\
\hline 13 & Vancouver & OTTAWA & Denver & Vancouver & New York & Vancouver & Montréal \\
\hline 14 & Grand Rapids & $\begin{array}{c}\text { Colorado } \\
\text { Springs }\end{array}$ & Philadelphia & Hamilton & Atlanta & Raleigh & Seattle \\
\hline 15 & Montréal & Richmond & New York & Minneapolis & Montréal & Nashville & Dallas \\
\hline 16 & Honolulu & Jacksonville & Columbus & Syracuse & Colorado Sp & Toronto & Orlando \\
\hline 17 & Portland & Albany & Hartford & Nashville & Minneapolis & Montréal & Atlanta \\
\hline 18 & Albany & Sarasota & Seattle & Rochester & Chicago & Boston & Toronto \\
\hline 19 & Boston & San Diego & Columbia & Montréal & Houston & Austin & Greenville \\
\hline
\end{tabular}

\section{Emplois (\%) dans le secteur des services professionnels, scientifiques et techniques}

Plus encore que dans la précédente, cette classe réunit des activités tertiaires qui traduisent ce que l'on en- tend spontanément par "économie du savoir». C'est ici que l'on retrouve la plupart des emplois dans toute la gamme des services qui touchent directement à l'informatique (design; programmation; production de logiciels, etc.) ainsi que les services proprement 
« scientifiques » consacrés à la recherche et au développement. C'est donc ce $6^{\mathrm{e}}$ indicateur qui, plus que les autres, témoignerait en principe de la spécialisation d'une ville en termes d'emplois dans ce que nous pouvons appeler l'économie du savoir, du moins pour le secteur tertiaire. D'entrée en jeu, notons que 5 des 9 RMR canadiennes se classent dans le « top $20 »$, Calgary étant en troisième position, Ottawa en quatrième, suivie de Toronto. Bref, les villes canadiennes s'en tirent très bien dans ce secteur représentatif de l'économie du savoir. Pour ce qui est des États-Unis, on y retrouve sans surprise les piliers du « high tech »: Boston, San Francisco, Raleigh et Austin. L'industrie des services professionnels, qui comprend des secteurs comme la comptabilité, la gestionconseil et la publicité, a tendance à se concentrer dans les plus grandes métropoles et les capitales, telles que New York, Chicago, San Francisco, Washington et Philadelphie. Quant à la bonne performance de Colorado Springs et d'Albuquerque, elle est le fruit des activités de recherche militaire.

\section{Emplois (\%) dans l'industrie de l'information et l'industrie culturelle ${ }^{20}$}

À la lecture du «top 20 » relatif à cet indicateur, il n'est pas étonnant d'y retrouver New York et Los Angeles (dont Hollywood). La présence de Nashville s'explique sans doute par son rôle dans ce que les Américains appellent l'Entertainment Industry. Les trois grandes villes canadiennes s'y trouvent elles aussi, ce qui confirme en quelque sorte leur statut de métropoles culturelles et informationnelles. Ottawa, pour sa part, est tout près, se classant au $22^{\mathrm{e}}$ rang. D'autres villes davantage associées aux activités high tech se retrouvent aussi dans le «top $20 »$ : Raleigh, Seattle, Boston, Austin, San Francisco et Denver. D'autres encore, comme Washington, Atlanta et Dallas, traduisent sans doute un mélange de fonctions culturelles, informationnelles et technologiques. La présence de villes comme Little Rock et Tulsa s'explique plus difficilement sans une connaissance plus poussée de leurs structures économiques.

\section{Les liaisons aériennes internationales (\%) ${ }^{21}$}

Il semble difficile d'établir un rapport entre les villes dont les aéroports affichent un pourcentage élevé de vols internationaux (hors de l'Amérique du Nord) et leur place dans l'économie du savoir selon les classements précédents. Cependant, cet indicateur a un rap- port étroit avec la taille urbaine. Par ailleurs, la localisation joue, de toute évidence, pour beaucoup. Les premières six villes sont toutes des villes côtières situées aux extrémités du continent. L'orientation géographique internationale de leur trafic aérien n'est donc pas un hasard. Par contre, une ville comme Chicago, située au milieu du continent, est davantage orientée vers les vols intercontinentaux, nonobstant la taille de son aéroport. Notons qu'Ottawa se classe au $35^{\mathrm{e}}$ rang. Longtemps mal desservie et dans l'ombre de Montréal qui est à moins de deux heures de route, l'aéroport d'Ottawa connait depuis environ cinq ans un essor sans précédant, alors que le nombre de passagers augmente à un rythme annuel de $10 \%$. Plusieurs vols directs sont maintenant offerts vers les principales villes américaines et européennes. Une nouvelle aérogare ultramoderne de 150 millions de dollars a également ouvert ses portes en octobre 2003 afin de répondre à cette croissance.

\section{Conclusion}

Sans pour autant retrouver au Canada l'équivalent des grands pôles intellectuels que sont les régions de Boston et de San Francisco (dont Sillicon Valley), les quatre plus grandes régions urbaines canadiennes se positionnent très bien au chapitre des indicateurs portant sur la scolarité et des mixes industriels. D'autre part, notre analyse indique clairement que le taux d'homicides par ville et le pourcentage de vols internationaux directs n'affichent aucune relation significative avec d'autres variables. Quant à Ottawa, elle peut sans doute être considérée comme une ville du savoir nord-américaine. Si l'on pense que 1) le classement sur le nombre de doctorats décernés en science et en génie par 1000 habitants ne tient pas compte des nouveaux programmes offerts depuis peu dans les universités d'Ottawa et Carleton, que 2) la région de la capitale canadienne réussit à se classer au $22^{\mathrm{e}}$ rang au chapitre du pourcentage d'emplois dans l'industrie de l'information, et que 3) l'aéroport d'Ottawa vient tout juste d'ouvrir une nouvelle aérogare afin de poursuivre son expansion rapide, il est possible de croire qu'Ottawa figurera dans tous les « top $20 »$ d'ici peu.

\footnotetext{
Quant à Ottawa, elle peut sans doute être considérée comme une ville du savoir nord-américaine.
} 


\section{Notes et références}

1 Rémy Tremblay est docteur en géographie de l'Université d'Ottawa. Il est présentement stagiaire postdoctoral à l'INRS-UCS. Il travaille dans le cadre de la Chaire de recherche du Canada en études urbaines et régionales sur les facteurs de localisation des villes du savoir en Amérique du Nord.

2 Gertler, M. S. et al. (2002). Competing on Creativity: Placing Ontario's Cities in North American Contex,. Toronto, Ontario Ministry of Enterprise, Opportunity and Innovation et Institute for Competitiveness and Prosperity.

3 Tremblay, R. (2002). La passion pour les classements. Revue des principaux "rankings" des technopoles américaines et canadiennes, Montréal, INRS-Urbanisation, Culture et Société.

4 Gertler, M.S. et al., op. cit.; Florida, R. (2001). The Economic Geography of Talent, Pittsburgh,Working Paper, Heinz School of Public Policy and Management, Carnegie Mellon University; Florida, R. (2002). The Rise of the Creative Class, New York, Basic Books.

5 Saxanian, A. (1994). Regional Advantage: Culture and Competition in Silicon Valley and Route 128, Cambridge, Harvard University Press; Mather, V. (1999). «Human Capital-based Strategy for Regional Economic Development », Economic Development Quarterly, vol. 13, p. 203216; Kotkin, J. et Devol, R. (2001). Knowledge-Value Cities in the Digital Age, Santa Monica, Milken Institute.

6 Diener, E. et Suh, E. (1997). « Measuring Quality of Life : Economic, Social, and Subjective Indicators », Social Indicators Research, vol. 40, p. 22-37; Kotkin, J. (2000). The New Geography, New York, Random House; Andrews, C.J. (2001). "Analyzing Quality-of-Place», Environment and Planning B, vol. 28, p. 201-217.

7 Parr, J. (1998). Detroit : Struggling against History, College Park, Academy of Leadership, University of Maryland.

8 Ghent Mallett, J. (2002). Silicon Valley North: The Formation of the Ottawa Innovation Cluster, Ottawa, Information Technology Alliance of Canada.

9 Tremblay, R. (2002), op. cit.

10 Miller, S. et Adams, S. (2001). Defining the British Columbia High Technology Sector Using. NAICS, Victoria, BC Stats \& Ministry of Competition, Science and Enterprise of British Columbia, p. 6 .

11 American Electronics Association et NASDAQ (2000). Cybercities. A City-by-City Overview of the High-Technology Industry, Washington, DC, American Electronics Association.
12 Cortright, J. et Meyer, H. (2001). High-Tech Specialization: A Comparison of High Technology Centers, Washington, DC, Center on Urban \& Metropolitan Policy, The Brookings Institution.

13 Devol, R.C. (1999). America's High-Tech Economy. Growth, Development, and Risks for Metropolitan Areas, Santa Monica, Milken Institute.

14 Atkinson, R.D. et Gotlieb, P.D. (2001). The Metropolitan New Economy Index. Benchmarking Economic Transformation in the Nation's Metropolitan Areas, Washington, Progressive Policy Institute.

${ }^{15}$ Markusen, A. et al. (2001). Gauging Metropolitan «HighTech » and "HI-Tech» Activity, Minneapolis, Humphrey Institute of Public Affairs, University of Minnesota, Working Paper 257.

16 Il s'agit du taux par 1000 habitants de meurtres (premier et second degrés), d'homicides volontaires et involontaires et d'infanticides. Parmi les indicateurs de criminalité, le taux d'homicides est le seul qui puisse être comparable entre le Canada et les États-Unis (Gannon, M., 2001. Feasibility Study on Crime Comparisons between Canada and the United States, Ottawa, Canadian Centre for Justice Statistics, Statistics Canada.). Les données américaines proviennent du FBI et sont pour les MSA seulement. Les données du FBI datent de 2001, sauf pour Albany (2000). Les données pour Chicago et St. Louis sont absentes puisque leur définition territoriale est différente des autres MSA. Les données canadiennes proviennent du recensement de Statistique Canada de 2001. Le taux pour Ottawa-Gatineau ne compte qu'Ottawa.

17 Les données sur l'éducation proviennent du US Census 2000 et de Statistique Canada 2001. Il s'agit des personnes de 15 ans et plus ayant au moins un baccalauréat. Les taux par 1000 sont divisés par la population totale, incluant les personnes de moins de 15 ans.

18 Le même commentaire s'applique à cet indicateur mais, cette fois-ci, il s'agit de diplômes de doctorat (Ph.D.). Cependant, il s'agit d'une définition plus restrictive du capital humain et qui se limite aux personnes les plus scolarisées.

19 Cet autre indicateur du capital humain porte sur les diplômes décernés et non pas sur les détenteurs de Ph.D. dans une ville, car ces derniers peuvent avoir étudié ailleurs. Il s'agit du nombre de personnes ayant obtenu un doctorat en sciences et en génie aux États-Unis en 2001 (National Science Foundation, 2001) et au Canada en 1998 (commande spéciale auprès de Statistique Canada). Les CMSA sont prises en compte, c'est-à-dire que, par exemple, les récipiendaires de $\mathrm{Ph} . \mathrm{D}$. en sciences et en génie en 2001 des universités de Standford à Palo Alto, Californie à Berkeley et de San Jose State à San Jose, entre autres, se 
réfèrent à la CMSA de San Francisco-Oakland-San Jose. Les taux par 1000 sont divisés par la population totale.

20 Grâce au SCIAN, il est possible d'établir des comparaisons entre le Canada et les États-Unis par secteur industriel détaillé. Cependant, les données américaines disponibles au moment de la rédaction de cet article (sur la base du recensement de 2000) se limitaient à des classes agrégées (deux chiffres). Le secteur manufacturier, par exemple, ne constituait qu'une seule classe. Les emplois classés selon le SCIAN (deux chiffres) actuellement disponibles pour les États-Unis permettent, cependant, une identification relativement détaillée des classes tertiaires (les services) plus conformes aux réalités de l'économie du savoir.
Nous avons retenu deux classes: les indicateurs 6 et 7 . Notre choix des industries qui forment l'économie du savoir se limite, par conséquent, au secteur tertiaire.

21 Cet indicateur nous informe sur le pourcentage de vols internationaux directs par rapport au trafic aérien total de tous les aéroports (lorsqu'il y en avait plus qu'un) d'une ville donnée. Par exemple, la donnée se rapportant à Montréal inclut Dorval et Mirabel. Les données américaines proviennent du U.S. Bureau of Transportation Statistics et sont pour l'année 2000. Elles excluent le Canada et le Mexique. Les données canadiennes proviennent du recensement de Statistique Canada de l'an 2000 et excluent les vols directs vers les États-Unis. 